Trauma Berufskrankh 2005 $\cdot 7[$ Suppl 1]:S148-S152 DOI 10.1007/s10039-004-0958-6

Online publiziert: 13. November 2004

๑) Springer Medizin Verlag 2004

K. Seide · U. Schümann · C. Jürgens · BG-Unfallkrankenhaus Hamburg

\title{
Klinische Erfahrungen mit dem computergesteuerten Hexapodfixateur
}

den klinischen Routineeinsatz entwickelt $[1,3]$. Ein manuell einzustellendes System wurde bisher an 127 Patienten für die Behandlung von Frakturen und Fehlstellungen eingesetzt. Als Erweiterungen des Systems wurden motorgetriebene [4] und den Kraftfluss dreidimensional messende Fixateure [6] als Labormuster entwickelt und in einzelnen klinischen Fällen erprobt.

\section{Methode}

\section{Prinzip}

Die parallele Kinematik [7] wird in der Robotertechnik für die räumliche Positionierung von Plattformen mit hoher Genauigkeit und Stabilität verwendet. Sie findet sich u. a. in Flugsimulatoren, bei denen über Hydraulikzylinder die Lage des
Cockpits geregelt wird. Das parallele kinematische System besteht aus 6 Linearantrieben („Hexapod“), welche an nicht blockierten Kugelgelenken fixiert sind. Wie bei entsprechenden Robotern üblich, können mit dem System beliebige Knochenbewegungen mit Präzision durchgeführt werden (• Abb. 1).

Für die Anwendung ist immer eine Software erforderlich. Sie implementiert die mathematischen Beziehungen zwischen den Knochen- und Ringbewegungen einerseits und den Längen der 6 Distraktoren andererseits. Verwendet wird ein IBM-kompatibler Personal Computer unter Windows [5].

Eine dreidimensionale Kraftflussmessung im Fixateur wurde mit 6 in den Hexapoden eingebrachten uniachsialen Kraftsensoren $(\bullet \mathrm{Abb} .2)$ realisiert.

einstellbares Fixateursystem auf der Ba-
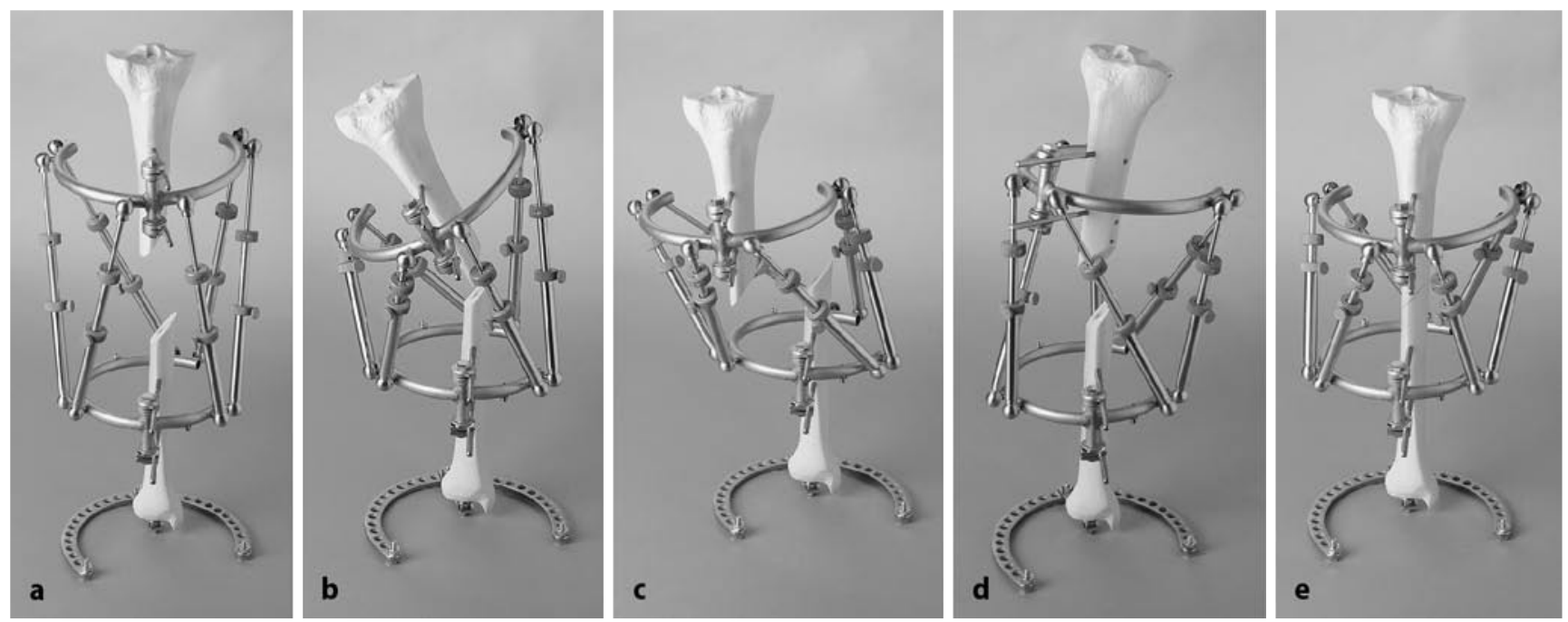

Abb. $1 \Delta$ Bewegungsmöglichkeiten des Hexapodfixateurs durch Längeneinstellung an 6 Distraktoren: a Verlängerung, b Achskorrektur, c Translation, d Torsion, e Grundstellung, neue, besonders einfach zu montierende 11-mm-Rohrringsysteme 

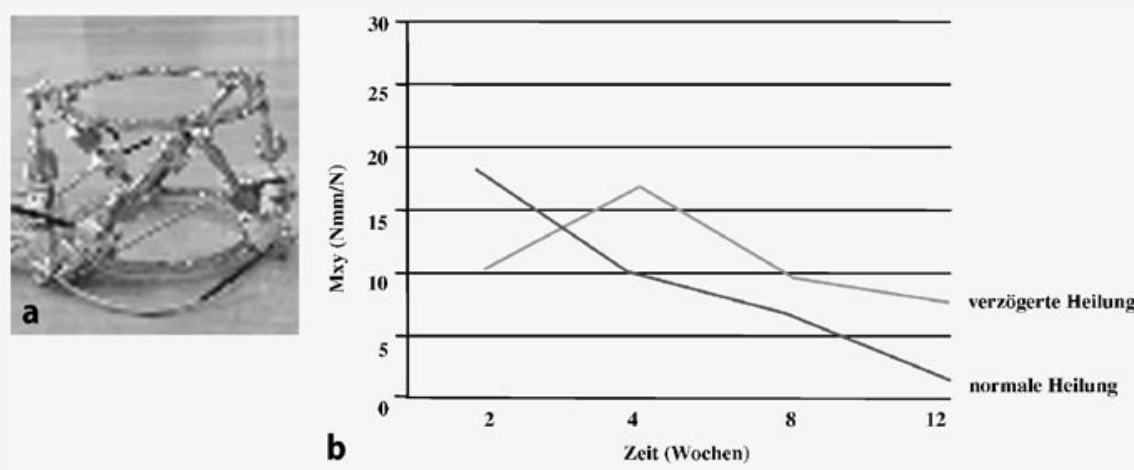

Abb. $2 \Delta$ Messfixateur (a) und gemessene Momente im Fixateur während der Knochenheilung für einen Patienten mit normalem und einen mit verzögertem Heilungsverlauf (b)

Abb. 3 Erweitertes Bewegungsausmaß der neuen Distraktoren mit gegenläufigen, teleskopisch ineinander laufenden Gewindestangen (rechts) gegenüber den herkömmlichen Distraktoren (links)
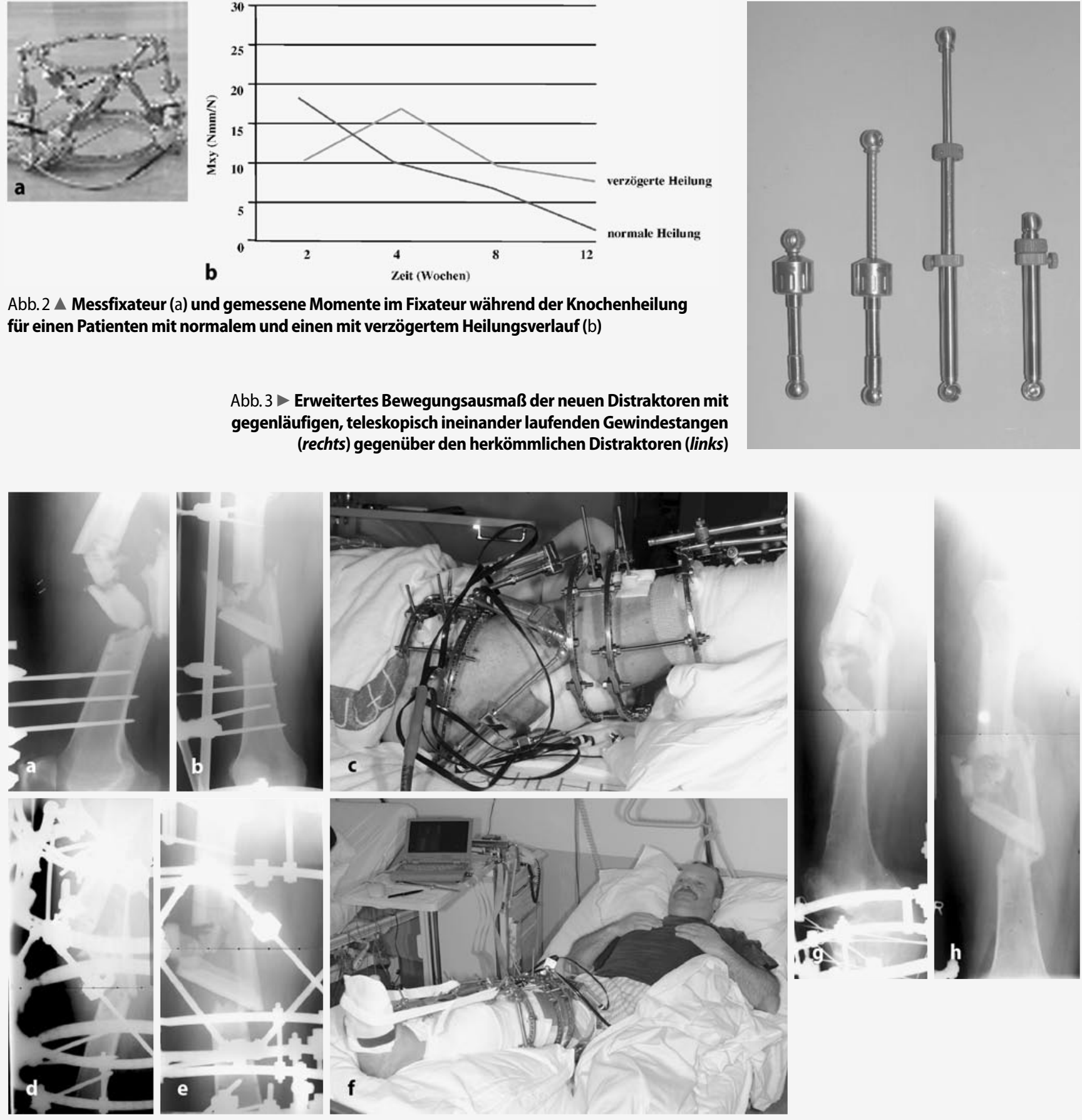

Abb. 4 Motorhexapodfixateur, sukzessive Reposition einer Translationsdislokation von $80 \mathbf{~ m m}$ mit Verkürzung am Oberschenkel, 5 Wochen nach Motorradunfall mit Komplexverletzung des gesamten Beins, a, b Ausgangsbefund, $c$, d, e klinisches Bild und Röntgen nach Montage des Motorhexapoden an die vorhandenen Schanz-Schrauben, $f$ Patient während der laufenden Reposition, g, h Ergebnis, Reposition erfolgte über 5 Tage

\section{Aktuelle Weiterentwicklung der Elemente}

Nachteile der ersten Systeme waren, dass bei größeren Bewegungsauslenkungen Distraktorelemente während der Behandlung ausgetauscht werden mussten und dass die verwendeten Lochringe die Montage erschwerten. Aufgrund der klinischen Erfahrungen wurden dann die Anwendung vereinfachende technische Lösungen erarbeitet.

Zum einen wurden Distraktorelemente mit einem großen linearen Bewegungs- ausmaß entwickelt (• Abb. 3). Diese bestehen aus 3 teleskopisch ineinander fahrenden, Gewinde tragenden Elementen. Aufgrund einer Kombination eines Rechtsmit einem Linksgewinde erfolgt durch Drehen des mittleren Elements eine Längenänderung. Eine konstruktive Besonder- 
Trauma Berufskrankh 2005 · 7[Suppl 1]:S148-S152

DOI 10.1007/s10039-004-0958-6

(C) Springer Medizin Verlag 2004

\section{K. Seide $\cdot$ U. Schümann $\cdot$ C. Jürgens}

\section{Klinische Erfahrungen mit dem computergesteuerten Hexapodfixateur}

\section{Zusammenfassung}

Auf der Basis einer parallelen Roboterkinematik wurde ein dreidimensional einstellbares Fixateursystem für den klinischen Routineeinsatz entwickelt. Wie bei entsprechenden Robotern üblich, können mit diesem Knochenbewegungen mit hoher Präzision durchgeführt werden. Ein manuell einzustellendes System wurde bisher an 127 Patienten für die Behandlung von Frakturen und Fehlstellungen eingesetzt. Unter Anwendung der entwickelten Software erfolgten Frakturrepositionen mit dem Hexapoden sukzessive und schmerzfrei, ggf. über eine Zeitspanne von mehreren Tagen. Im Ilisarov-Fixateur hat sich das System ins-

besondere bei Korrekturen in Kombination mit einer Distraktion bewährt. Elektromotorische Antriebe wurden bei 4 Patienten eingesetzt. Ein den Kraftfluss dreidimensional messender Fixateur wurde als Grundlage für einen „intelligenten“ Fixateur erprobt. Dieser wird in Zukunft eine automatisch kontrollierte Fakturbehandlung oder Fehlstellungskorrektur auf Basis des Hexapodfixateurs ermöglichen.

\section{Schlüsselwörter}

Hexapodroboter · Fraktur · Fehlstellung ·

Computerassistierte Chirurgie .

Fixateur externe

\section{Clinical experience with the computer-controlled hexapod fixator}

\begin{abstract}
Hexapod robot kinematics have been exploited to develop an external fixator that is adjustable in all six spatial degrees of freedom. As with other robot systems, bones can be moved with a high degree of precision. A manually controlled fixator has so far been used with appropriate software in 127 patients. Secondary fracture reduction was performed successively, if necessary over several days, without causing pain to the patients. llizarov's system for combined correction and distraction was especially useful.
\end{abstract}

heit ist, dass lange Gewinde innerhalb der Rohre mit jeweils kurzen endständigen Außengewinden gepaart sind. Dadurch werden sichtbare Gewinde vermieden.

Zum anderen wurden neue Ringsysteme konstruiert. Diese bestehen aus 11-mmRohren, welche zu einem 2/3-Kreis gebogen wurden. An diese sind sowohl die Gelenke für den Hexapodmechanismus als auch Längsträger und Backen für die Schanz-Schrauben angeschweißt. Eine Erweiterung mit Elementen anderer 11-mmRohrfixateure ist möglich.

Die neuen Distraktoren ermöglichen eine Verstellung von $105 \mathrm{~mm}$ auf $235 \mathrm{~mm}$, d. h. auf 224\% der Ausgangslänge. Die neuen Ringsysteme lassen eine besonders schnelle Montage zu. Aufgrund der geschweißten vorgegebenen Konstruktion sind lediglich die Schanz-Schrauben einzubringen und deren Backen festzuziehen.

\section{Motorfixateur}

Statt der Distraktoren sind 6 elektrische Linearantriebe (Escap 23LT2R mit Getriebe 1:10o und optoelektronischen Winkelschrittgebern für die Positionskontrolle) montiert (• Abb. 4).

Über eine Elektronik sind die Motoreinheiten mit einem Notebook-Computer verbunden. Für die klinische Anwendung wurde eine spezielle Steuersoftware entwickelt. Nach Eingabe der Fixateurgeometrie und einer gewünschten Bewegung steuert der Computer die Motoren in die errechnete Position.

Die Motorelemente sind zu entsprechenden manuellen Distraktoren kompatibel, durch Schnappmechanismen der Kugelgelenke kann in Abhängigkeit von den Erfordernissen der Behandlung ein einfacher Austausch erfolgen. Für die Durchführung der Röntgenkontrollen wurde dieser Austausch jeweils vorgenommen, da die derzeitigen, noch recht großen Motoren zu einer Überlagerung mit der Frakturzone führten. Der Motorhexapodfixateur wurde bisher in 4 Fällen klinisch erprobt.

\section{Software}

Es existieren 2 Versionen. Zum einen für die Anwendung im Ilisarov-Fixateur, zum anderen für die Anwendung am neuen 11mm-Rohrfixateur. 

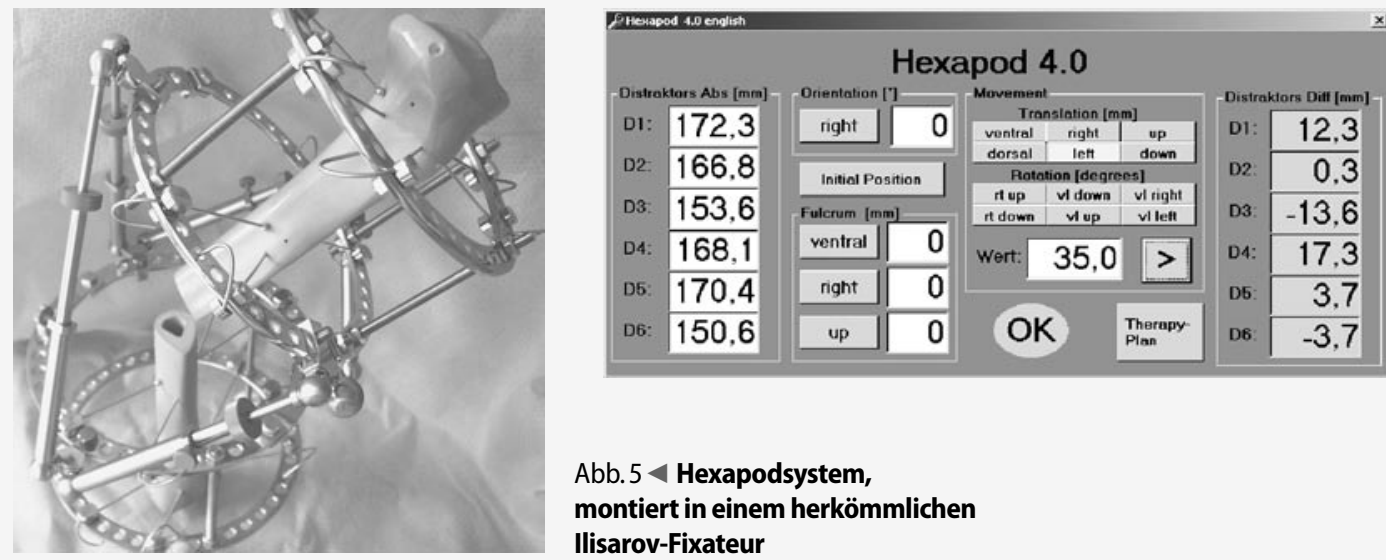

Abb. $6<$ Softwaremaske

für den 11-mm-

Rohrringfixateur

Abb. $5<$ Hexapodsystem,

montiert in einem herkömmlichen

Ilisarov-Fixateur
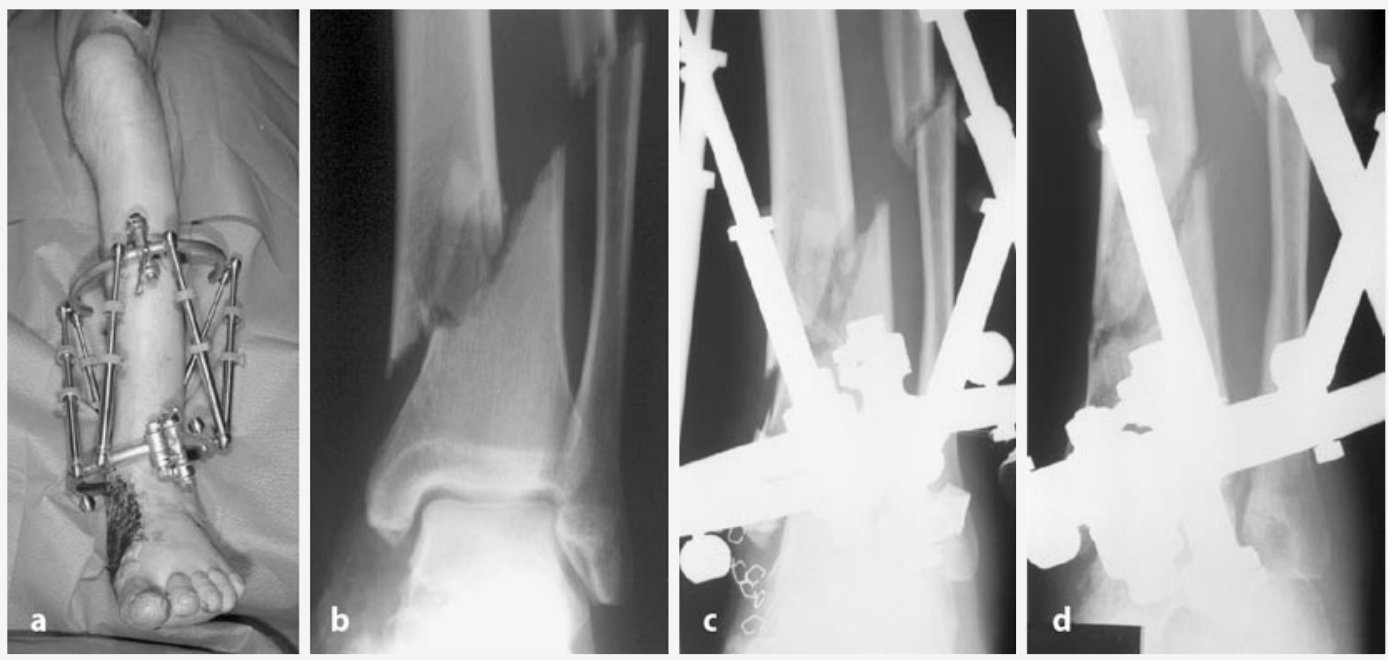

Abb. $7<$ Sukzessive schmerzfreie sekundäre Frakturreposition, a klinisches Bild, b Röntgenbilder nach Unfall, c am 1. postoperativen Tag, d am 3. postoperativen Tag

Beim Ilisarov-Fixateur kann die Montage an vorhanden Konstruktionen und mit wenigen Einschränkungen an beliebigen freien Ringlöchern erfolgen (• Abb. 5). Das Programm [5] hierfür besteht aus den 2 alternativen Bildschirmmasken „Absolut“ und „Differenz“. Mittels der Maske „Absolut" wird die Fixateurmontage erfasst. Die Ringdurchmesser, die Gelenkpositionen auf den Ringen und die Längen der 6 Distraktoren werden eingegeben. Aus diesen Angaben wird die aktuelle Position des oberen Rings gegenüber dem unteren Ring errechnet. In der Maske „Differenz" werden die für eine vorgegebene Knochenbewegung erforderlichen Einstellungen der 6 Distraktoren berechnet. Für die Berechnung werden ein Drehpunkt (CORA) und die Größe und Richtung der Korrekturbewegungen eingegeben. Es lassen sich beliebige Verfahrwege des Knochens realisieren.

Für den Rohrfixateur ergibt sich aufgrund der fest montierten Gelenke eine wesentlich einfachere Maske (• Abb. 6).
Ergebnisse

\section{Frakturbehandlung}

Der Hexapod ermöglicht ein neues Behandlungskonzept bei der Frakturbehandlung. Im Rahmen der notfallmäßigen Fixateuranlage wird eine schnelle Reposition durchgeführt, dabei jedoch auf eine Feinreposition verzichtet. Hierdurch ergibt sich eine wesentliche Zeitersparnis, welche bei einem kritischen Gesamtzustand für den Patienten wichtig ist.

Gelegentlich ist aufgrund der Spannungen in den Weichteilen ein sofortiger vollständiger Längenausgleich geschlossen nicht möglich. Auf grobe Dehnungs- und Einrenkungsmanöver kann in solchen Fällen verzichtet werden. Die exakte Reposition erfolgt unter Anwendung der Software mit dem Hexapoden sukzessive, ggf. über eine Zeitspanne von mehreren Tagen (• Abb. 7). Dieses Vorgehen könnte als „computerassistierte minimalinvasive Frakturreposition“ bezeichnet werden.
Eine genaue Reposition erleichtert auch das Umsteigen auf innere Osteosyntheseverfahren, z. B. unter Verwendung von winkelstabilen internen Fixateursystemen, die über kleine Inzisionen eingebracht werden. Repositionsmanöver erfolgten bei stabilen Bedingungen und sind deshalb für den Patienten schmerzfrei.

\section{Korrekturen im Ilisarov-Fixateur}

Durch Anwendung des Hexapoden sind beliebige vorgegebene Knochenbewegungen mit einer einfachen und stabilen Konstruktion präzise durchzuführen. Insbesondere sind Translationen, Achskorrekturen und Torsionen in Kombination mit der Distraktion ohne komplizierte Konstruktionen zu realisieren. Das System bewährte sich als vorteilhafte Möglichkeit zur Korrektur, insbesondere bei schwierigen posttraumatischen oder postinfektiösen Zuständen [2]. Auch Abweichungen bei linearer Distraktion mit einer Standardkonstruktion konnten durch nachträgliche Montage des 

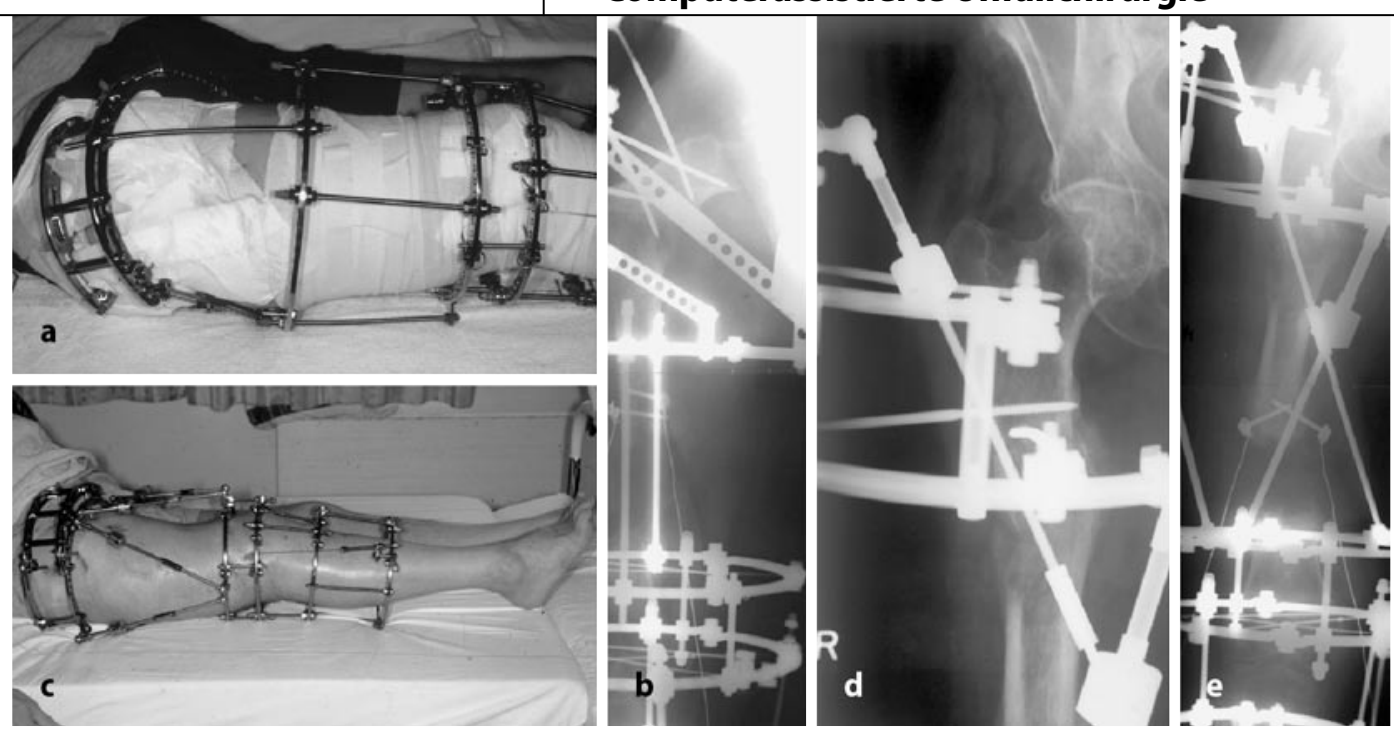

Abb. $8<$ Korrektur einer zunehmenden Abkippung des proximalen Oberschenkelfragments $\left(70^{\circ}\right)$ während einer bifokalen Verlängerung/Segmenttransport durch Weichteilspannung. Montage und Röntgenbilder vor $(a, b)$ und nach $(c, d, e)$ der sukzessiven Korrektur
Hexapoden in den liegenden Fixateur korrigiert werden (• Abb. 8)

\section{Motorfixateur}

Er wurde bisher in 4 Fällen klinisch erprobt [4]. Auch hier war die Schmerzfreiheit der Reposition durch die langsame Durchführung, auch bei großen Dislokationen ( $\bullet$ Abb. 4), besonders beeindruckend.

\section{Diskussion}

\section{Schlussfolgerung}

Zusammen mit den neuen Bauteilen bietet der Hexapodfixateur die Möglichkeit, mit wenigen Handgriffen in sehr kurzer Zeit eine Frakturstabilisierung zu erreichen, wobei die Reposition, ggf. auch sekundär, punktgenau erfolgen kann.

Im Ilisarov-Fixateur ergaben sich besondere Vorteile bei der Kombination einer Distraktion mit einer Korrektur einer komplexen Fehlstellung, insbesondere auch, wenn während einer Distraktion unvorhergesehene Abweichungen auftreten.

\section{Zukünftige Weiterentwicklung: „Intelligenter" Fixateur}

Unter Verwendung moderner Mechanik und Elektronik erscheint es durch Kombination der Motorsteuerung mit einer zusätzlichen Lastmessung (• Abb. 2, [6]) und optimierter wissensbasierter Software möglich, einen sich selbstständig an die Heilungssituation anpassenden und den Chirurgen bei verschiedenen Manövern wie Frakturrepositionen, Korrekturen und sukzessiven Knochenneubildungen (Distraktionsosteogenese) - auch unter komplexen Nebenbedingungen - unterstützenden Fixateur externe zu realisieren. Ein solches System wird einen ultimativen zukünftigen Standard der externen Knochenbehandlung darstellen.

In einem vom BMBF geförderten Projekt wird derzeit - auf der Grundlage bereits mechanisch realisierter Teilfunktionalitäten - ein elektronisch gesteuerter ,intelligenter" Fixateur externe entwickelt, welcher den Arzt durch folgende Funktionalitäten unterstützen kann:

1. automatische Frakturreposition durch dreidimensionale Fixateurbewegungen

2. automatische dreidimensionalen Lastmessung mit Steuerung der Belastung im Kallus

3. frühzeitige Erkennung von Heilungsproblemen

4. automatische sukzessive Kallusdistraktion

Eine darüber hinaus vorgesehene Kombination mit aktuellen telemedizinischen Verfahren wird eine weitere Optimierung des Heilungsverlaufs bei maximaler Sicherheit für den Patienten und wahrscheinlicher Kostenreduktion durch Vermeiden von Arztbesuchen ebenso wie durch die ambulante Früherkennung von Problemen ermöglichen.

\section{Korrespondierender Autor PD Dr. K. Seide}

BG-Unfallkrankenhaus Hamburg, Bergedorfer Straße 10, 21033 Hamburg

E-Mail:k.seide@buk-hamburg.de

Interessenkonflikt: Keine Angaben

\section{Literatur}

1. Seide K, Wolter D (1996) Universelle dreidimensionale Korrektur und Reposition mit dem Ringfixateur unter Anwendung der Hexapod-Anordnung. Unfallchirurg 6: 422-424

2. Seide K, Wolter D (2000) Korrekturen mit dem He xapoden. Orthopäde 29: 39-46

3. Seide K, Wolter D, Kortmann HR (1999) Fracture reduction and deformity correction with the hexapod Ilizarov fixator. Clin Orthop 363: 186-195

4. Seide K, Schümann U, Jürgens Ch (2002) Erste klinische Erfahrungen mit einem elektromotorisch einstellbaren Fixateur externe. Trauma Berufskrankh 4: $427-430$

5. Seide K, Wollnack J, Weinrich N et al. (2002) Theorie und Software des Hexapod Fixateurs externe. Biomedizinische Technik 2002

6. Seide K, Wolter D, Jürgens C et al. (2002) Is X-ray free monitoring of fracture healing possible by multidimensional load measurements in an external fixator? Transactions of the $48^{\text {th }}$ Annual Meeting of the Orthopaedic Research Society, Poster No. 0717

7. Stewart $D$ (1965) A platform with six degrees of freedom. Proc Inst Mech Eng 180: 371-378 